\title{
09
}

\section{Эффект фотовращения Квинке}

() А.И. Грачев

Физико-технический институт им. А.Ф. Иофффе РАН, Санкт-Петербург, Россия

E-mail: grach.shuv@mail.ioffe.ru

Поступило в Редакцию 19 марта 2018 г.

Предложен способ реализации аналога известного эффекта вращения Квинке, который может быть назван фотовращением Квинке. Эффект основан на ранее рассмотренном автором явлении вращения в постоянном электрическом поле сферической частицы в условиях ее непрерывного освещения, индуцирующего электрический дипольный момент.

DOI: 10.21883/PJTF.2018.16.46473.17303

В работе [1], в которой впервые было рассмотрено явление вращения в постоянном электрическом поле сферической частицы в условиях непрерывной фотогенерации ее электрического дипольного момента (фотоиндуцированное вращение - ФИВ), высказано предположение, что одновременное проявление ФИВ и известного эффекта - вращения Квинке (ВК) [2-5] — мало вероятно. Однако данное предположение не совсем корректно. Действительно, при выполнении соотношения $\sigma_{p}>>\sigma_{l}$ (здесь $\sigma_{p}$ и $\sigma_{l}-$ электрическая проводимость частицы и окружающей ее жидкой среды соответственно), которое является оптимальным для ФИВ, проявление ВК невозможно (см. рисунок, $a$ ). Однако при противоположном соотношении $\sigma_{p}<\sigma_{l}$ (точнее, $\tau_{p}>\tau_{l}$, где $\tau_{p}$ и $\tau_{l}$ - максвелловское время релаксации для частицы и жидкой среды соответственно), реализуемом в случае ВК (см. рисунок, $b$ ), эффект ФИВ также может наблюдаться, хотя при этом неизбежно снижение величины фотоиндуцированного дипольного момента $\mathbf{P}_{p h}$. Более того, поскольку ФИВ в отличие от ВК проявляется при любой величине внешнего электрического поля $\mathbf{E}_{0}$, оно в чистом виде может реализоваться до значений $E_{0} \leqslant E_{c}$ (где $E_{c}-$ величина критического поля для ВК), а в области более высоких полей оба механизма вполне способны работать одновременно. Следует отметить, что при реализации данного сценария спонтанный характер ВК в отношении 


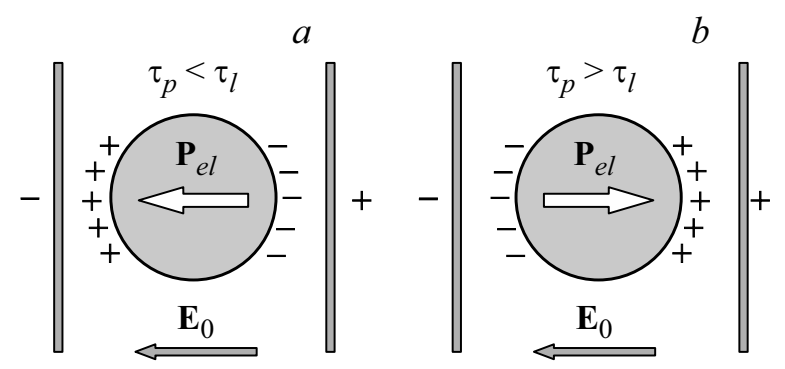

C

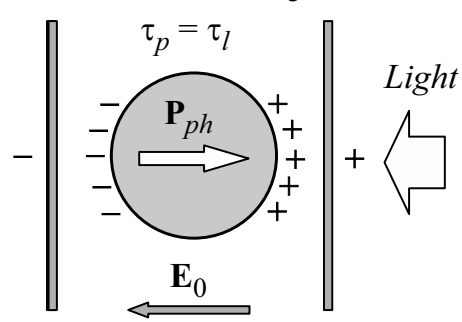

Распределение поверхностного заряда и ориентация электрического дипольного момента сферы, отвечающие устойчивому положению сферы $(a)$, условию вращения Квинке $(b)$, условию фотовращения Квинке при предположении отсутствия электрически индуцируемого поверхностного заряда $(c)$.

направления и знака вектора угловой скорости вращения $\omega$ будет отсутствовать. Таким образом, реализуется один из возможных вариантов оптического управления (синхронизации) ВК ансамблем частиц (что является предметом отдельной публикации).

Как показано в настоящей работе, механизм ФИВ обеспечивает еще одну любопытную возможность: практически полное его совпадение с ВК в плане особенностей экспериментального проявления последнего, a именно спонтанности $\omega$, необходимости превышения $E_{0}$ некой величины, а также наличия зависимости $\omega \sim E_{0}^{2}$. Поэтому данное явление с полным основанием может быть названо фотовращением Квинке (ФВК).

Для наиболее эффективного проявления ФИВ требуется, чтобы вектор $\mathbf{P}_{p h}$ был ортогонален $\mathbf{E}_{0}$, поскольку по мере увеличения (уменьшения) угла $\alpha=\pi / 2 \pm \delta$ между указанными векторами величина кру-

Письма в ЖТФ, 2018, том 44, вып. 16 
тящего момента будет снижаться. В случае адиабатического вращения, удовлетворяющего условию [1]

$$
\omega_{p h} \tau_{p} \ll 1,
$$

где $\omega_{p h}$ - угловая скорость стационарного вращения, величина последней не зависит от знака $\delta$. Однако для режима неадиабатического вращения указанная симметрия нарушается, поскольку теперь следует учитывать, совпадает ли проекция $\mathbf{P}_{p h}$ на направление вектора $\mathbf{E}_{0} \mathrm{c}$ направлением электрически индуцируемого дипольного момента сферы $\mathbf{P}_{e l}$ или противоположна ему. Это обусловлено тем, что в случае указанного режима сопротивление вращению будет оказывать не только момент $\mathbf{T}_{l i q}$, воздействующий на сферу со стороны вязкой жидкости, но и $\mathbf{P}_{e l}$ (точнее, эффективный дипольный момент, зависящий от $\omega[3]$ ). Ясно, что при сложении или вычитании указанной проекции векторов $\mathbf{P}_{p h}$ и $\mathbf{P}_{e l}$ степень торможения сферы должна быть различной, т.е. значения стационарной скорости вращения будут различаться. В целом будет различаться и динамика вращения, которая будет определяться исходным значением $\delta$, величиной $\mathbf{T}_{l i q}$, а также конкретным видом зависимости от $\omega$ оптически и электрически индуцируемых моментов сферы. Однако наиболее ярко обсуждаемая асимметрия проявится в случае коллинеарности $\mathbf{P}_{p h}$ и $\mathbf{E}_{0}$, сопровождающейся их совпадением или противоположной ориентацией, поскольку первый случай отвечает устойчивому состоянию сферы (см. рисунок, $a$ ), а во втором она может оказаться в условиях неустойчивого равновесия (см. рисунок, $b$ ), которое при определенных экспериментальных условиях может вызвать вращение сферы, т. е. эффект ФВК.

Рассмотрим экспериментальный случай, когда для сферической частицы (радиуса $R$ ), освещаемой со стороны одного из электродов, и окружающей ее жидкости выполняется следующее условие (см. рисунок, $c$ ):

$$
\varepsilon_{l} \sigma_{p}-\varepsilon_{p} \sigma_{l}=0,
$$

где $\varepsilon_{l}$ и $\varepsilon_{p}$ - диэлектрическая проницаемость жидкости и частицы соответственно. Как указывалось выше, при выполнении условия (2) ВК частицы исключено, что позволяет без нарушения общности более наглядно продемонстрировать физику эффекта ФВК. Действительно, в этом случае поверхностный заряд $Q_{r}=\left|-Q_{r}\right|=\left|+Q_{r}\right|$, обусловленный диэлектрической поляризацией и проводимостью сферы и

Письма в ЖТФ, 2018, том 44, вып. 16 
жидкости, будет равен нулю, а следовательно, отсутствует и связанный с ним дипольный момент сферы (отметим, что здесь подразумевается случай относительно малых значений $\omega$, когда дисперсией величин, входящих в (2), можно пренебречь).

На рисунке, $c$ направление распространения светового пучка, как и $\mathbf{P}_{p h}$, коллинеарно вектору $\mathbf{E}_{0}$, однако направления этих векторов противоположны. Нетрудно понять, что данная геометрия отвечает экспериментальным условиям наблюдения ВК (см. рисунок, $b$ ), и поэтому для описания поведения частицы в указанных экспериментальных условиях может быть использован теоретический аппарат, разработанный для ВК [5]. Как и в случае последнего, любой случайный поворот сферы вокруг оси, ортогональной $\mathbf{E}_{0}$, ведет к появлению вращающего момента, стремящегося развить возникшее отклонение. Перерастание случайного поворота сферы в ее устойчивое вращение требует превышения величиной $E_{0}$ значения критического поля $E_{c}^{p h}$, которое теперь будет определяться следующим выражением:

$$
E_{c}^{p h}=\left(E_{0} \gamma / P_{p h} \tau_{p}\right)^{1 / 2}=E_{0}\left(\omega_{p h} \tau_{p}\right)^{-1 / 2},
$$

где $\gamma$ - коэффициент трения вращения сферы в данной жидкой среде, а $\omega_{p h}=P_{p h} E_{0} \gamma^{-1}$ - введенная выше угловая скорость стационарного адиабатического вращения в том случае, когда $\mathbf{P}_{p h} \perp \mathbf{E}_{0}$ [1]. Как следует из $(3), E_{c}^{p h} / E<1$, если $\omega_{p h} \tau_{p}>1$, при этом для скорости стационарного вращения ФВК $\omega_{p q}$ может выполняться условие $\omega_{p q} \tau_{p} \ll 1$, что следует из выражения для случая ВК $[3,4]$

$$
\omega_{p q}=\tau_{p}^{-1}\left[\left(E_{0} / E_{c}^{p h}\right)^{2}-1\right]^{1 / 2}=\tau_{p}^{-1}\left[\omega_{p h} \tau_{p}-1\right]^{1 / 2}
$$

Видно, что при незначительном превышении произведением $\omega_{p h} \tau_{p}$ единицы величина $\omega_{p q} \tau_{p}$ действительно может быть сколь угодно малой.

Поскольку потеря устойчивости сферы связана с воздействием случайных факторов, направление вектора $\omega$ имеет спонтанный характер (при выполнении условия ортогональности оси вращения $\mathbf{E}_{0}$ ). Однако в отличие от ВК в данном случае имеется очевидная возможность управления вращением, для чего достаточно задать небольшой начальный угол между векторами $\mathbf{P}_{p h}$ и $\mathbf{E}_{0}$. Тогда в области полей $E_{0}<E_{c}^{p h}$ должно наблюдаться хотя и относительно слабое, но устойчивое ФИВ, которое начнет трансформироваться в ФВК после достижения величины $E_{c}^{p h}$. 
Ясно, что этот механизм управления ФВК близок к описанному выше способу оптического управления вектором $\omega$ частиц в случае ВК.

Ясно, что нарушение в ту или иную сторону условия (2) не повлияет на возможность реализации $Ф В К$, если остается выполненным неравенство

$$
Q_{r}<Q_{p h}
$$

где $Q_{p h}=\left|-Q_{p h}\right|=\left|+Q_{p h}\right|-$ фотогенерируемый заряд сферы. В противном случае могут быть реализованы два сценария: сохранение устойчивого положения сферы либо эффект фотостимуляции ВК, который прежде всего выразится в снижении величины критического поля $E_{c}$ (естественно, сохраняется и возможность указанного выше способа контроля направления $\omega$ ).

В заключение рассмотрим интересный вопрос, связанный с реализацией ФВК в том случае, когда окружающая частицу среда обладает бесконечно малой вязкостью. Конкретно, это может быть случай сферы, левитирующей в вакууме. Поскольку условие (2) экспериментально нереализуемо, для наблюдения ФВК требуется выполнение неравенства (5), что легко достигается путем выбора необходимого значения $E_{0}$, с учетом того, что теперь $E_{c}^{p h}=0$. Динамика движения сферы после начала ее спонтанного (или заданного) вращения будет описываться уравнением

$$
J(d \omega / d t)=T_{r}(\omega),
$$

где $J-$ момент инерции сферы, $T_{r}(\omega)$ - эффективный вращательный момент с учетом электрически индуцируемого дипольного момента. В отсутствие сопротивления со стороны окружающей среды угловая скорость частицы будет непрерывно расти вне зависимости от конкретного вида $T_{r}(\omega)$. Ясно, что подобный сценарий не может отвечать физической реальности, и поэтому возникает вопрос: каков механизм установления стационарного вращения и какой величины может достигать стационарная угловая скорость? На данный момент можно предложить один из вариантов развития событий: для частиц с $R \sim 10 \mathrm{~nm}-10 \mu \mathrm{m}$ при достижении значений $\omega$, превосходящих $10^{11}-10^{8} \mathrm{~s}^{-1}$, будет превзойден предел удельной прочности материала частиц, что вызовет их механическое разрушение. В принципе указанный вариант может быть использован для экспериментальных исследований прочности частиц микронных и субмикронных размеров в сравнении с макроскопическими образцами того же материала. Следует

Письма в ЖТФ, 2018, том 44, вып. 16 
также отметить, что реализация столь высоких скоростей вращения может обеспечить экспериментальную базу для наблюдения недавно предсказанного латерального эффекта Казимира [6].

\section{Список литературы}

[1] Грачев А.И. // ФТТ. 2018. Т. 60. В. 4. С. 666-669.

[2] Quincke G. // Ann. Phys. Chem. 1896. V. 59. N 11. P. 417-486.

[3] Jones T.B. // IEEE Trans. Ind. Appl. 1984. V. IA-20. N 4. Pt 1. P. 845-849.

[4] Turku I. // J. Phys. A: Math. Gen. 1987. V. 20. N 11. P. 3301-3308.

[5] Jones T.B. // Electromechanics of particles. Cambridge, N.Y.: Cambridge University Press, 1995. 265 p.

[6] Manjavacas A., Rodríguez-Fortuño F.J., García de Abajo F.J., Zayats A.V. // Phys. Rev. Lett. 2017. V. 118. N 13. P. 133605. 